\title{
Some new trends of intelligent simulation optimization and scheduling in intelligent manufacturing
}

\author{
Xinyu $\mathrm{Li}^{1} \cdot$ Chunjiang Zhang ${ }^{1}$
}

Published online: 17 July 2020

(c) Springer-Verlag London Ltd., part of Springer Nature 2020

\section{Introduction}

Intelligent manufacturing has become a major trend in the development of the manufacturing industry around the world and is being studied and applied by numerous industrially developed countries, including USA, Germany, Japan and so on. The intelligent simulation optimization and scheduling techniques play important roles in intelligent manufacturing. They directly impact production efficiency, and manufacturing costs and profit.

Taking advantage of virtual simulation technology, simulation optimization is used for pre-planning in production line layout, plant resource allocation and scheduling, production process, warehouse and logistics, etc. Based on the rehearsal of the simulation model, some problems or shortcomings can be found by analysis, evaluation and verification. Adjustment and optimization can be done in time to reduce the number of changes and rework of the physical system in the subsequent production execution links, thereby effectively reducing costs, shortening the construction period and improving efficiency. Scheduling can be defined as "allocating limited resources to tasks in time to meet or optimize one or more goals." Since 1990s, scheduling theory and methods have been the focus of international research in the field of manufacturing systems.

Xinyu Li

lixinyu@mail.hust.edu.cn

1 State Key Laboratory of Digital Manufacturing Equipment and Technology, School of Mechanical Science and Engineering, Huazhong University of Science and Technology, Wuhan, China

\section{Future trends of intelligent simulation optimization and scheduling}

With the rapid development of a new generation of information technology (such as cloud computing, Internet of Things, big data, mobile Internet, artificial intelligence, etc.) and its continuous in-depth applications in the manufacturing field, the era of intelligent manufacturing is coming. Simulation optimization and scheduling in intelligent manufacturing has made fruitful achievements both in theory and practice $[1,2]$. However, there are still some possible research prospects in this field worthy to be studied in the future.

- Combination with reinforcement learning

Reinforcement learning is an important category of methods in machine learning. Agents in reinforcement learning learn strategies to maximize returns or achieve specific goals during the interaction with the environment. Like simulation optimization and scheduling, reinforcement learning is essentially a decision-making process for optimization purposes, which is a new research hotspot in the field of artificial intelligence. It has achieved good results in a closed environment like games [3]. The rules in the environment of games are very clear, and it is easy to sample a large number in this environment so that reinforcement learning can get good results. However, in the real manufacturing environment, due to a large amount of uncertainties, high sampling cost and long sampling time, deep reinforcement learning is difficult to use. A very intuitive idea is to build virtual simulation models for sampling. Some scholars have already made some explorations in this direction [4]. It is promising to construct simulation models for some manufacturing environment and take reinforcement learning for scheduling, especially for online scheduling.

- Human modeling in simulation and scheduling 
Although scheduling has been studied for many years, the classic scheduling problems contain some harsh assumptions. Therefore, the successful application of scheduling requires a relatively high degree of standardization and automation. Standardization and automation are not difficult for machines. However, human factors still exist in most current manufacturing systems, and human-machine collaboration system [5] is also one of the future development directions of manufacturing. Compared with machines, human behavior, psychology, emotions and other factors are much more complicated. In the current research, scheduling usually only considers tasks and processing machines, not human factors or simply models workers like machines. The current model for the human is not practical in the actual production, resulting in deviations in the scheduling plan during execution. In the future, human psychology, behavior, emotion and other factors should be modeled and the mutual relationship between human behavior and manufacturing system should be considered. Based on this, the simulation and scheduling will be more practical.

\section{- Data-driven simulation and scheduling}

Dynamic events such as machine failures, urgent insertion of orders, changes in delivery dates and changes in orders will seriously affect the stability, efficiency of production. How to quickly respond to the dynamic events in the workshop environment to ensure the efficient and stable execution of the scheduling plan has always been one of the key problems in the workshop schedule. Due to the extensive use of smart devices and smart sensors in workshop production, the data generated during the manufacturing process can be fully collected. Using data to better serve workshop production has become a current research hotspot. Introducing data analysis methods in workshop scheduling, accurately predicting dynamic events such as machine failures and building a new data-driven workshop dynamic scheduling model to achieve fast and accurate responses and processing of dynamic events is one research focus on schedule in the future.

\section{- Scheduling in cloud manufacturing}

As a new manufacturing model, cloud manufacturing can provide on-demand manufacturing services to consumers through the Internet. Scheduling is one of the key technologies to realize the cloud manufacturing. The scheduling in cloud manufacturing can be divided into platform level, enterprise level and workshop level. Platform-level scheduling refers to the cloud platform operators analyzing and decomposing the manufacturing tasks submitted by consumers. According to the characteristics of tasks, quality requirements, execution progress and the current status of different resource providers, the tasks are reasonably allocated to enterprises. Enterprise-level scheduling is to assign cloud tasks to different workshops. Workshop-level scheduling refers to the scheduling of machines, machining centers and other resources. Among them, the scheduling of cloud platform needs to consider the real-time production status of different enterprises or workshops; at the same time, enterprises or workshops may have tasks that are not from the cloud platform, so enterprises or workshops need to consider the status of cloud platform in order to adjust the priority of production tasks or other strategies to maximize benefits. Therefore, cloud platform scheduling and enterprise or workshop scheduling need collaborative optimization to achieve better scheduling and improve the efficiency of cloud manufacturing.

\section{- Scheduling in digital twin shops}

The digital twin is one of the hottest topics in intelligent manufacturing [6]. The goal of intelligent manufacturing is to integrate and use a new generation of information technology to build smart factories and carry out smart production to meet socialization, personalization, service, intelligence, green and other manufacturing development needs and trends. One of the bottlenecks to achieve this goal is how to realize the interaction and fusion between the physical world and the cyber world of manufacturing. Intelligent simulation and scheduling will play an increasingly important role in the digital twin shops. The new generation of production system simulation needs to be integrated with MES system, artificial intelligence and other technologies. It is physically connected to the actual production system through various API interfaces. It uses data-driven simulation models, stores and manages online data and provides AI for analysis. AI uses production system simulation to perform enhanced previews to form decision judgments and then downloads the program to automatically adjust the actual production system to form a closed loop for intelligent manufacturing systems.

\section{- Simulation and scheduling in unmanned factories}

Since the outbreak of the COVID-19, new demands have been placed on the application of unmanned technology. Under the long-term accumulation of technology, unmanned applications using various intelligent robots as carriers have gradually shown their power. Unmanned factories are the ultimate vision of manufacturing. With the development of robot technology, information technology and artificial intelligence technology, intelligent simulation optimization and scheduling technology is bound to be an important part of unmanned factories. Unmanned factories require very mature simulation technology for verification. Scheduling 
technology in an unmanned factory will not only consider the processing of workpieces, but also need to consider the logistics of the entire process, the connection of various processing links and the treatment of defective products, etc.

In future, we believe that more and more intelligent simulation and scheduling works will emerge in all aspects of manufacturing and play more and more important roles in the coming era of intelligent manufacturing.

\section{References}

1. Zhang L, Zhou L, Ren L, Laili Y (2019) Modeling and simulation in intelligent manufacturing. Comput Ind 112:103123

2. Gao K, Huang Y, Sadollah A, Wang L (2020) A review of energyefficient scheduling in intelligent production systems. Complex Intell Syst 6(2):237-249
3. Silver D, Hubert T, Schrittwieser J, Antonoglou I, Lai M, Guez A, Hassabis D (2018) A general reinforcement learning algorithm that masters chess, shogi, and Go through self-play. Science 362(6419): 1140

4. Shi J-C, Yu Y, Da Q, Chen S-Y, Zeng A-X (2019) Virtual-taobao: virtualizing real-world online retail environment for reinforcement learning. Proc AAAI Conf Artif Intell 33:4902-4909

5. Wang L, Gao R, Váncza J, Krüger J, Wang XV, Makris S, Chryssolouris G (2019) Symbiotic human-robot collaborative assembly. CIRP Ann 68(2):701-726

6. Tao F, Qi Q (2019) Make more digital twins. Nature 573:490-491

Publisher's Note Springer Nature remains neutral with regard to jurisdictional claims in published maps and institutional affiliations. 